\title{
Design and mechanical characterization of solid and highly porous 3D printed poly(propylene fumarate) scaffolds
}

\author{
Jason M. Walker ${ }^{1,2} \cdot$ Emily Bodamer $^{1} \cdot$ Alex Kleinfehn $^{3} \cdot$ Yuanyuan Luo $^{3}$. \\ Matthew Becker ${ }^{3} \cdot$ David Dean $^{1}$
}

Received: 28 December 2016 / Accepted: 14 March 2017 / Published online: 2 May 2017

(C) Springer International Publishing Switzerland 2017

\begin{abstract}
The primary goal of tissue engineering is to repair a defect by encouraging new tissue growth and remodeling of that tissue within a biodegradable scaffold. The scaffold is the centerpiece of tissue engineering efforts, and its design and properties are of paramount importance. The architecture of the scaffold will directly impact its mechanical strength, degradation characteristics, and capacity to guide neotissues into the defect. Scaffold porosity is frequently used as a solitary description of architecture, while feature dimensions such as strut size and pore size are largely ignored. It is well known that pore size and shape influence tissue regeneration while strut size (i.e., wall thickness) dramatically affect mechanical strength and resorption kinetics. In this work, we propose a methodology that places special emphasis on feature dimensions using a mathematical approximation of Schoen's gyroid, a triply periodic minimal surface, as the foundation for pore architecture. By modulating the gyroid and making virtual measurements of the resulting structures, we establish important relationships between feature dimensions and the governing equation. Using this foundation, scaffolds with gyroid-type porosity were designed and 3D printed out of a bioresorbable poly(propylene fumarate)-based resin. Unconfined compression testing was conducted on fully
\end{abstract}

David Dean

David.Dean@osumc.edu

1 Department of Plastic Surgery, The Ohio State University, 460 West 12th Ave., Rm. 388, Columbus, OH 43210, USA

2 Department of Mechanical and Industrial Engineering, Youngstown State University, 1 University Plaza, Youngstown, OH 44555, USA

3 Department of Polymer Science, The University of Akron, 170 University Ave Akron, Akron, OH 44325-3909, USA dense parts, as well parts with designed porosity, to establish a baseline for mechanical properties. Orientation-based mechanical anisotropy was seen in these 3D printed porous specimens. Finally, we demonstrate how such architectures can be embedded into anatomical shapes.

Keywords Tissue engineering · Scaffold design . Biofabrication - Additive manufacturing $\cdot 3 \mathrm{D}$ printing

\section{Introduction}

Tissue engineering (TE) is a rapidly evolving biotechnology field that aims to reconstruct and regenerate tissue lost to trauma or disease [1]. One of the most important aspects of TE is the design and fabrication of the scaffold [2]. TE scaffolds provide a temporary platform to hold bioactive molecules and facilitate the growth of neotissue. An ideal scaffold should have a porous structure which guides tissue formation and be composed of a biodegradable material that can safely resorb inside the body [3]. Other attributes which need to be addressed with regard to the design of TE scaffolds include biocompatibility, mechanical properties, and degradation rate. These last three attributes are, at least partially, dependent on the inner architecture of a porous scaffold. Conventional and subtractive methods for fabricating TE scaffolds and medical devices (e.g., molds for porous particulate materials, salt leaching or other porogens, and electrospun weaves) allow for some control over porosity and pore size but prohibit fine control of scaffold geometry [4]. Scaffolds with stochastic porosity lack repeatability in fabrication, potentially contain isolated void spaces, and present issues involving anisotropic degradation, tissue ingrowth, and stress distribution. 
Many TE researchers are turning to additive manufacturing (AM), also referred to as 3D printing, technologies to fabricate complex scaffolds designed in Computer Aided Design (CAD) software [5-8]. Using these methodologies, pore geometry is not dependent on the stochastic spatial distribution of pores or porogens; instead, pore geometry can be designed and printed. Indeed [9-11], these new manufacturing technologies are driving an increasing amount of research on pore geometry. In general, the size and shape of pores within the scaffold must balance mechanical integrity with bioactive molecule delivery, tissue infusion, and degradation characteristics [5, 12]. Pores should be gently curving and minimally tortuous so as not to hinder cell in-migration $[13,14]$. They should also be interconnected to encourage vascularization [15] and highly permeable to permit the inflow of nutrients, bioactive molecules, and disposal of metabolic waste [16]. Issues of flow in a scaffold are relevant both in vivo and perhaps even more so where shear forces may occur during in vitro (e.g., bioreactor) pre-culturing of a TE scaffold. Finally, the pore design should be easily created and rendered in the 3D modeling software (i.e., CAD software) and scaffold fabrication setup and monitoring software (i.e., Computer Aided Manufacture or CAM software).

Non-intersecting, triply periodic minimal surfaces (TPMS) offer an attractive foundation for the design of smooth, curved, interconnected pore networks [17-19]. One TPMS in particular, the gyroid (also known as Schoen's G surface), was presented by Alan Schoen in 1970 [20]. Since then, gyroid structures have been found to occur naturally in many different systems including block copolymers [21, 22], butterfly wing scales [23, 24], and cell membranes $[25,26]$. The gyroid surface can be described implicitly by a relatively simple nodal equation [27]. The surface itself separates 3D space into two complex and intertwined, but separate, phases. Subsequently, one phase is established as a solid network of struts, while the second phase is void and constitutes the porous volume. Due to their mathematical complexion, gyroid-type porous structures are extremely manipulable and computationally efficient.

In this paper, we demonstrate an algorithm for creating gyroid-type porous structures with user-defined pore size, strut size, and/or percent porosity based on Schoen's gyroid triply periodic minimal surface. A primary advantage of this method is that it provides the engineer/clinician the ability to define exact pore and strut sizes. Often, too much emphasis is placed on percent porosity when quantifying the geometry of TE scaffolds, while feature dimensions are largely ignored. Figure 1 illustrates the inadequacy of using porosity alone as a measure of scaffold geometry. Full control over the pore and strut dimensions makes it easier to tune scaffolds for optimal tissue ingrowth, mechanical
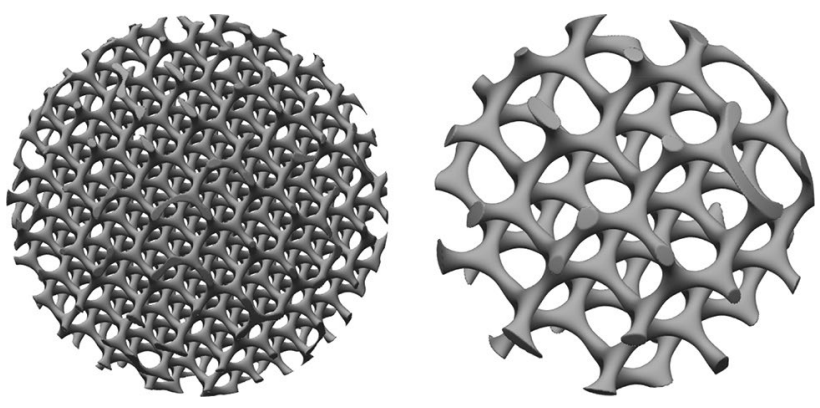

Fig. 1 Comparison of two different porous spherical architectures with the same external dimensions (i.e., diameter) and the same percent porosity

properties, and degradation characteristics. We will show in this study that the gyroid surface can be used to create a wide range of porous configurations ranging from approximately $2-98 \%$ porous.

\section{Porous scaffold design}

\subsection{Level surfaces}

The level set method is a tool for representing a closed curve using a level set function, $\Phi$. In two dimensions, the level set function takes negative values inside the curve and positive values outside. The level set method can be expanded to three dimensions, whereby the level set function is used to represent a closed surface and takes negative values inside the surface and positive values outside. Values that lie directly on the surface take the value zero and form the zero level set. Using this method, $\Phi$ is defined at every point in a space, not just on the surface, leading to the creation of a 3D scalar field. At each point, the function specifies a scalar value which indicates whether the point is inside, outside, or on the surface:

$\Phi(x, y, z)-\mathrm{C}= \begin{cases}<0, & \text { inside } \\ 0, & \text { on } \\ >0, & \text { outside. }\end{cases}$

For example, a sphere is represented implicitly by the zero level set of the algebraic function:

$\Phi_{\text {Sphere }}=x^{2}+y^{2}+z^{2}-r^{2}$

where $r$ is the radius of the sphere. Figure 2 displays a twodimensional scalar field for $\Phi_{\text {Sphere }}$ at $z=0$, with $\mathrm{r}=3.5$ and within the bounds $x=y=[-4,4]$. The surface of the sphere is constructed on the zero level set of $\Phi_{\text {Sphere }}$; that is, everywhere $\Phi_{\text {Sphere }}=0$. All of the points inside of the surface have negative values, while all of the points outside of the surface are positive. 


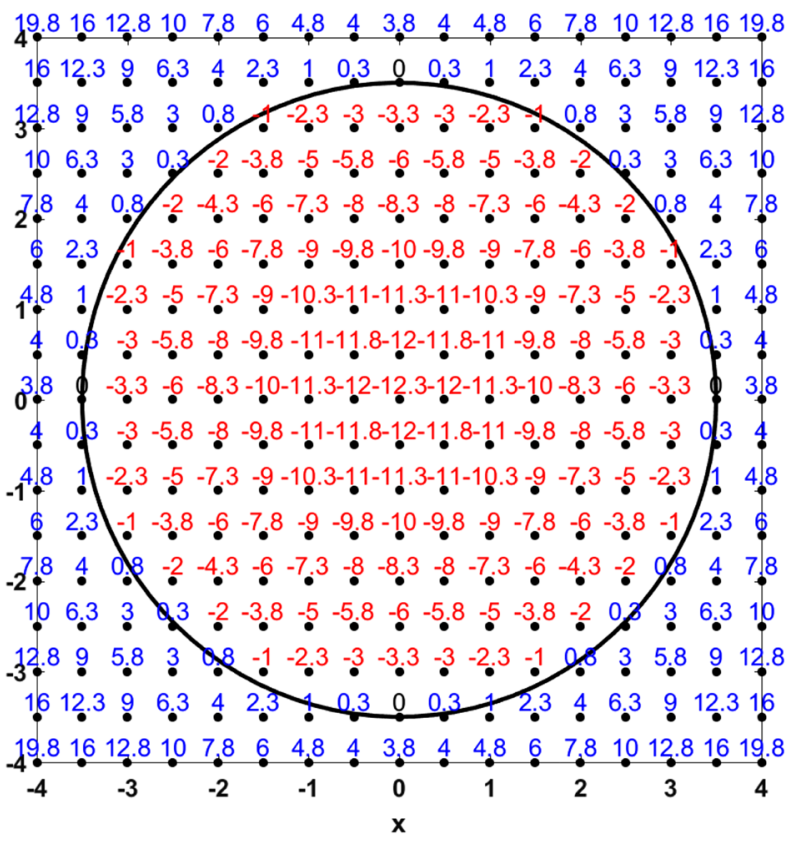

Fig. 2 Two-dimensional slice of the surface of a sphere represented by the zero level set with $r=3.5$ at $z=0$ in algebraic scalar field $\Phi_{\text {Sphere }}$

\subsection{The gyroid surface}

Schoen identified gyroid surfaces as belonging to a family known as triply periodic minimal surfaces [20]. The gyroid can be described implicitly, to the first order of approximation, by the nodal equation [27]:

$\Phi_{G}=\sin (N x) \cos (N y)+\sin (N z) \cos (N x)+\sin (N y) \cos (N z)-C$.

The gyroid surface is only truly minimizing when the level constant, $C$, is zero. In this case, the mean curvature everywhere on the surface becomes zero. With non-zero values of $C$, the mean curvature also becomes non-zero but remains constant over the entire surface. Using this approximation, the mean curvature loses its constancy [28] but such an effect bears no consequence in the development of TE scaffolds. Figure 3 displays a two-dimensional scalar field for $\Phi_{G}$ at $z=0$, with $C=0.67, N=0.67 \pi$ and within the bounds $x=y=[-4$, 4]. The surface of the gyroid is constructed on the zero level set, $\Phi_{G}=0$.

\subsection{Combining implicit surfaces}

Boolean operations can be used to combine two or more scalar fields. For example, the sphere in Fig. 2 can be made porous with pore architecture derived from the gyroid in

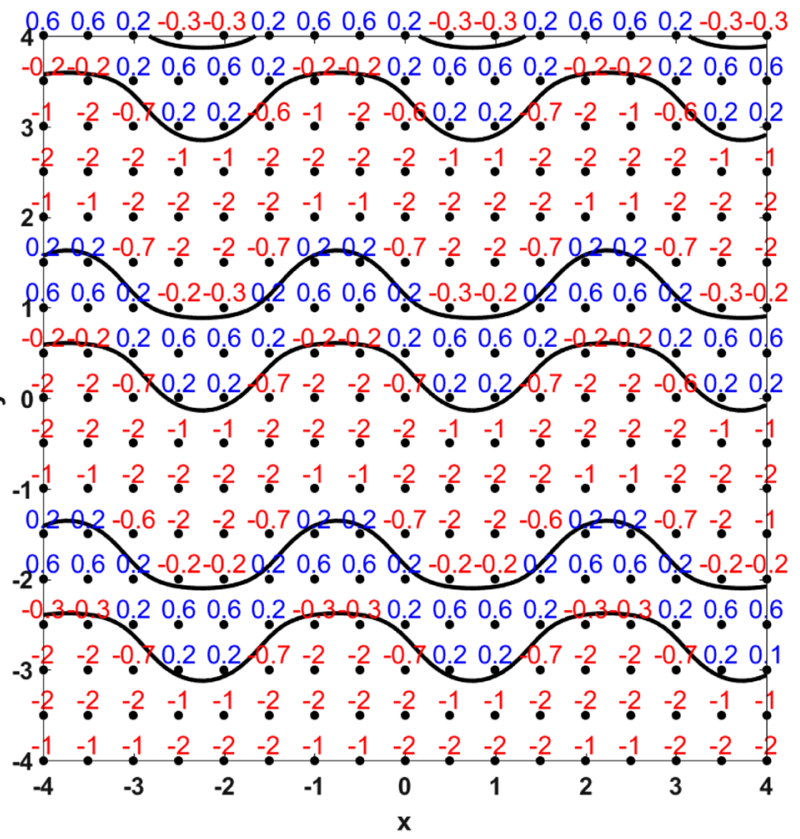

Fig. 3 Two-dimensional slice of the surface of a gyroid represented by the zero level set with $C=0.67$ and $N=0.67 \pi$ at $z=0$ in algebraic scalar field $\Phi_{G}$

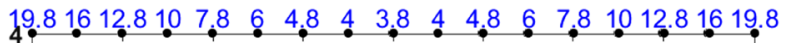

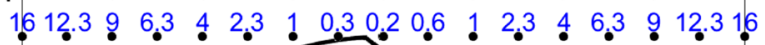

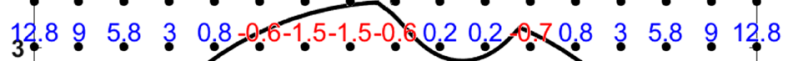

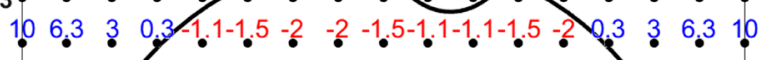

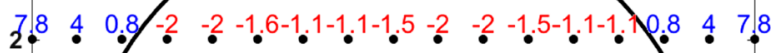

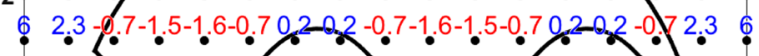
$4.8 \quad 1.2-0.2-0.3020 .60 .60 .2-0.3-0.20 .20 .60 .60 .2] 1.8$ $4.0 .30 .20 .60 .60 .2=0.2-0.20 .20 .60 .60 .2=0.20 .20 .20 .64$ $>0.8 .0 .0 .20 .20 .7-1.5-1.5-0.0 .20 .2,0.7-1.5-1.5-0.60 .23 .8$ $403-1.6-1.1-1.1-1.5-2.2-2-1.5-1.1-1.1-1.5-2-2-1.50034$

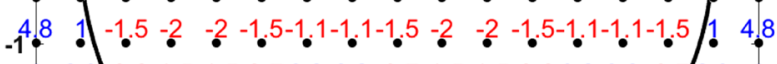

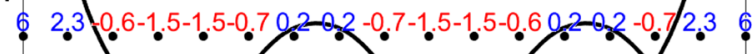
$-2.8400-0.2-0.20 .20 .60 .602-0.2-0.20 .20 .60 .60 .847 .8$ $106.3 \quad 3 \quad 0.60 .60 .2=0.3-0.30 .20 .60 .60 .2=0.3-3.3 \quad 3 \quad 6.310$

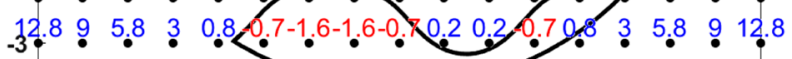

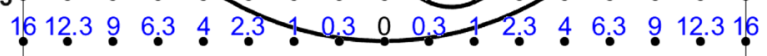

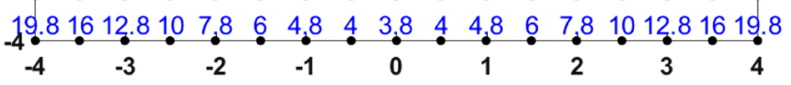

Fig. 4 Two-dimensional slice of the surface of a sphere with gyroidtype porous architecture represented by the zero level set at $z=0$ in algebraic scalar field $\Phi_{\text {Sphere } G}$

Fig. 3 by taking the maximum scalar value at each point and reconstructing the surface along the new zero level set (Fig. 4): 


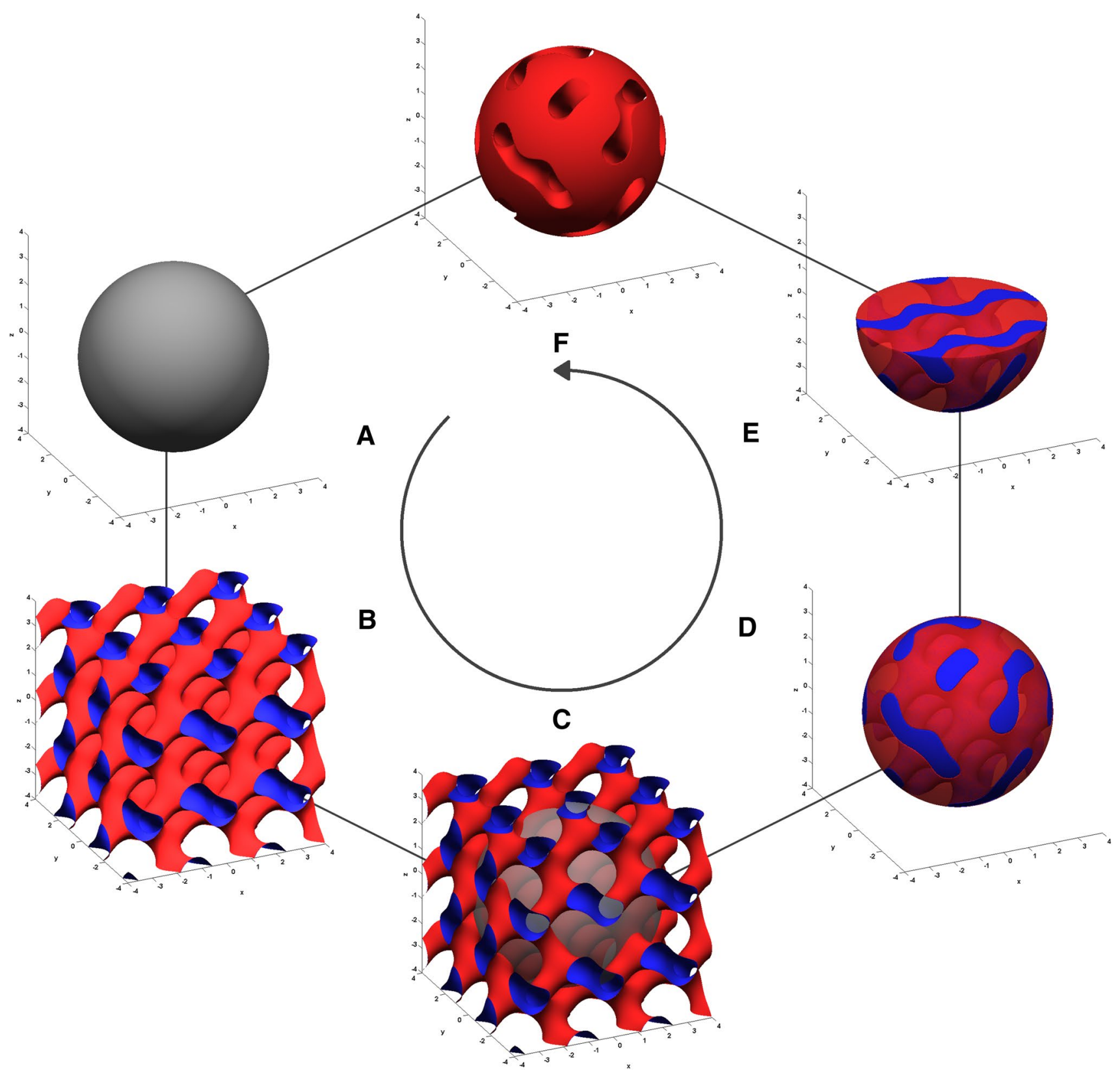

Fig. 5 Illustration of the process of combining surfaces using their scalar fields in three dimensions (see inline text for explanation)

$\Phi_{\text {Sphere }_{G}}=\max \left(\Phi_{\text {Sphere }}, \Phi_{G}\right)$.

Figure 5 illustrates the process of combining surfaces using scalar fields in three dimensions: (A) a sphere with radius $=3.5$ [analogous to Fig. 2]; (B) a gyroid surface with its inside colored red and its outside colored blue [analogous to Fig. 3]; (C) an overlay of a gyroid surface and a sphere; (D) a sphere partitioned into two separate phases by a gyroid surface; (E) a cutaway of that partitioned sphere at $\mathrm{z}=0$ [analogous to Fig. 4]; and (F) a sphere with a gyroid pore architecture after the blue phase is voided. This could be done with virtually any shape implant, including the arbitrary shape of a defect site-specific scaffold.

\subsection{Signed distance fields for arbitrarily-shaped objects}

The object to be made porous, $\Phi_{\text {object }}$, can be a shape defined by an implicit function (such as a sphere, cylinder, ellipsoid, etc.), but this is not a requirement. It is possible to use an arbitrarily-shaped object, such as CT-derived bone anatomy. Since there is no governing equation to describe 
the surface of an arbitrarily-shaped object, the scalar field must be determined manually. In this case, we implemented a custom algorithm in MATLAB® (MathWorks, Natick, MA) to calculate the signed distance field of a polygonal mesh. This algorithm iterates through every point in a bounded space and determines its distance to the surface, as well as whether the point is inside, outside, or on the surface [29]. Points inside of the surface are assigned negative distance values, while points outside of the surface remain positive and points on the surface are equal to zero by definition. The surface of the object which was discretely defined by a polygonal mesh becomes implicitly described by the zero level set of its signed distance field.

The signed distance field is a special case of a scalar field where each value gives the exact geometric distance to the zero level set. This is different than the scalar fields described in Sects. 2.1, 2.2, and 2.3 which give the algebraic distance to the zero level set. However, since both types of scalar fields capture their surfaces implicitly as zero level sets, they can be used interchangeably within the methods described here.

\subsection{Modulating the gyroid architecture}

The period of the gyroid surface can be modulated analogously to a simple sine or cosine wave. For example, with a period coefficient $N=2 \pi$, the gyroid surface is periodic in all three directions with a period, or unit side length, of 1 . By scaling the period, the size and number of pores can be tailored.

The gyroid can be further modulated by changing the level constant, $\mathrm{C}$. When $C=0$, the surface divides space into two congruent domains, each with a $50 \%$ volume fraction of the space. By adding or subtracting a nonzero constant, C, from the scalar field, the zero level set is shifted and porosities ranging from approximately 2-98\% can be obtained. Figure 6 demonstrates how varying $C$ changes the geometry and porosity, $n$, of the gyroid structure.

\subsection{Porosity, pore size, and strut size}

Porosity, $n$, is expressed as a decimal fraction in terms of the solid volume, $V_{\mathrm{S}}$, and the total volume, $V_{\mathrm{T}}$, where the total volume constitutes the sum of the solid and porous volumes:

$n=\left(1-\frac{V_{\mathrm{S}}}{V_{\mathrm{T}}}\right)$.

Another important parameter for describing the gyroid is the ratio of the pore dimension, $d_{\mathrm{p}}$, to the strut dimension, $d_{\mathrm{s}}$, and is represented by $\xi$ :

$\xi=\frac{d_{\mathrm{p}}}{d_{\mathrm{s}}}$

This ratio is of particular interest, because like porosity, it is a function of the level constant, $C$, independent of the period coefficient, $N$, which makes it easy to draw direct relationships between the implicit equation and the resultant geometry.

In the absence of a closed-form solution, these dimensions were determined by virtual measurements. The pore and strut dimensions were measured along the space diagonal [ $\left[\begin{array}{lll}1 & 1 & 1\end{array}\right]$ of a unit cell of the porous gyroid structure (Fig. 7). The unit cell was constructed by installing the gyroid architecture with $N=2 \pi$ in a unit cube with bounds $x=y=z=[0,1]$ and level constant, $C_{n}$, using the level set method previously described. To achieve high accuracy, a grid size of $749 \times 749 \times 749$ was used. A cubic grid with odd dimensions is required to have scalar values that lie directly on the space diagonal.

The marching cube algorithm was used to extract a polygonal mesh of the isosurface from the scalar field [30]. For porosity calculations, the solid volume is the volume enclosed by the polygonal mesh and was calculated using the divergence theorem [31], while the total volume is equal to the unit side length cubed.

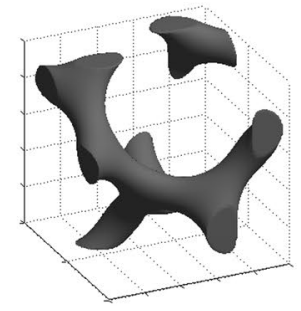

$n: \quad 90 \%$

C: $\quad-1.196$

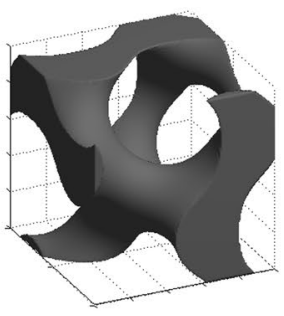

$70 \%$

$-0.617$

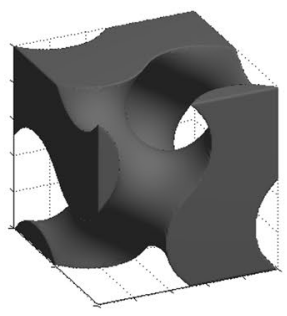

$50 \%$

0.000

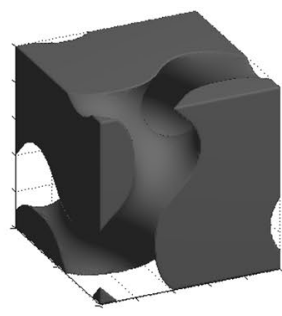

$30 \%$

0.617

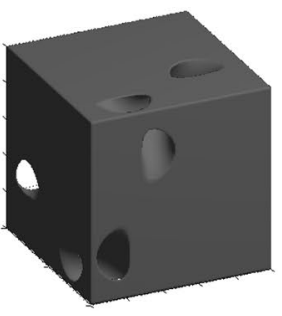

$10 \%$

1.196

Fig. 6 Illustration of the effect of the level constant, $C$, on the geometry and porosity, $n$, of the gyroid unit cell 


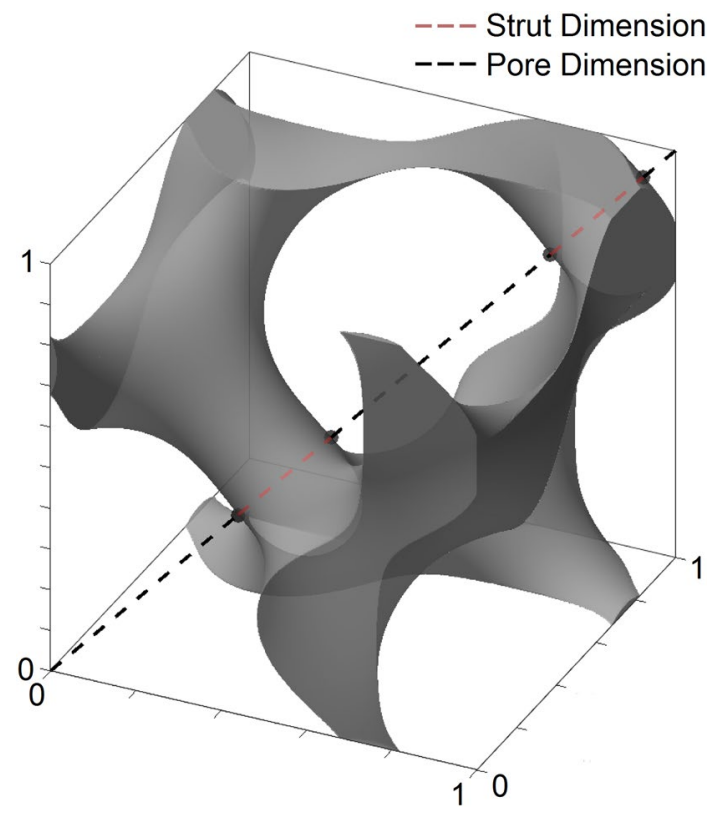

Fig. 7 Illustrations of the pore and strut dimensions of the gyroid unit cell along the space diagonal [ $\left[\begin{array}{lll}1 & 1 & 1\end{array}\right]$

\subsection{Computation of parameters}

Using a polynomial fit $\left(R^{2}=1.0000\right)$, a direct relationship is drawn between the level constant, $C$, and porosity (as a decimal fraction), $n$ (Fig. 8):

$C=0.7864 n^{3}-1.1798 n^{2}-2.5259 n+1.4597$.

Alternatively, the level constant can be calculated from the ratio of the pore dimension to the strut dimension, $\xi$ $\left(R^{2}=0.9996\right.$; Fig. 8$)$ :

$$
\begin{aligned}
C= & -0.0006 \xi^{5}+0.0162 \xi^{4}-0.1722 \xi^{3}+0.9142 \xi^{2} \\
& -2.5329 \xi+1.7889 .
\end{aligned}
$$

Once the level constant has been determined, it can be used to help calculate the appropriate period coefficient,

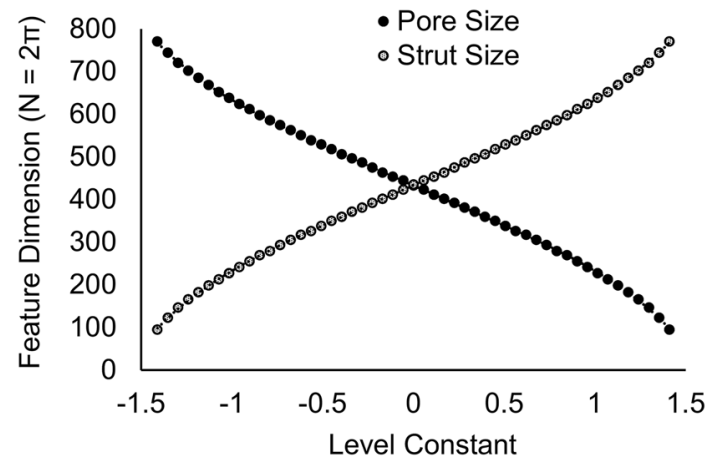

Fig. 9 Pore and strut dimensions as a function of level constant for the gyroid shapes with polynomial fits

$N$. First, the level constant is related to the pore and strut dimensions (independently) for the period coefficient, $N_{0}=2 \pi$ (Fig. 9). The following models give the pore and strut dimensions, respectively, as a function of $C$ with $N_{0}=2 \pi$ (both $\left.R^{2}=0.9999\right)$ :

$$
\begin{aligned}
d_{\mathrm{p}}^{2 \pi}= & -11.7311 C^{5}-0.1307 C^{4}-1.7987 C^{3}+0.2070 C^{2} \\
& -186.9928 C+433.0114 \\
d_{\mathrm{s}}^{2 \pi}= & 11.7311 C^{5}-0.0466 C^{4}+1.7987 C^{3}+0.0175 C^{2} \\
+ & 186.9928 C+433.0937 .
\end{aligned}
$$

The pore and strut dimensions scale linearly by $N$. Therefore, the output of either model can be related to the appropriate desired value, $d_{\mathrm{p}}$ or $d_{\mathrm{s}}$, to calculate a scale factor, $\gamma$ :

$\gamma=\frac{d_{\mathrm{p}}^{2 \pi}}{d_{\mathrm{p}}}=\frac{d_{\mathrm{s}}^{2 \pi}}{d_{\mathrm{s}}}$

which can then be used to determine the proper period coefficient, $N$ :

$N=\gamma N_{0}=\gamma 2 \pi$.
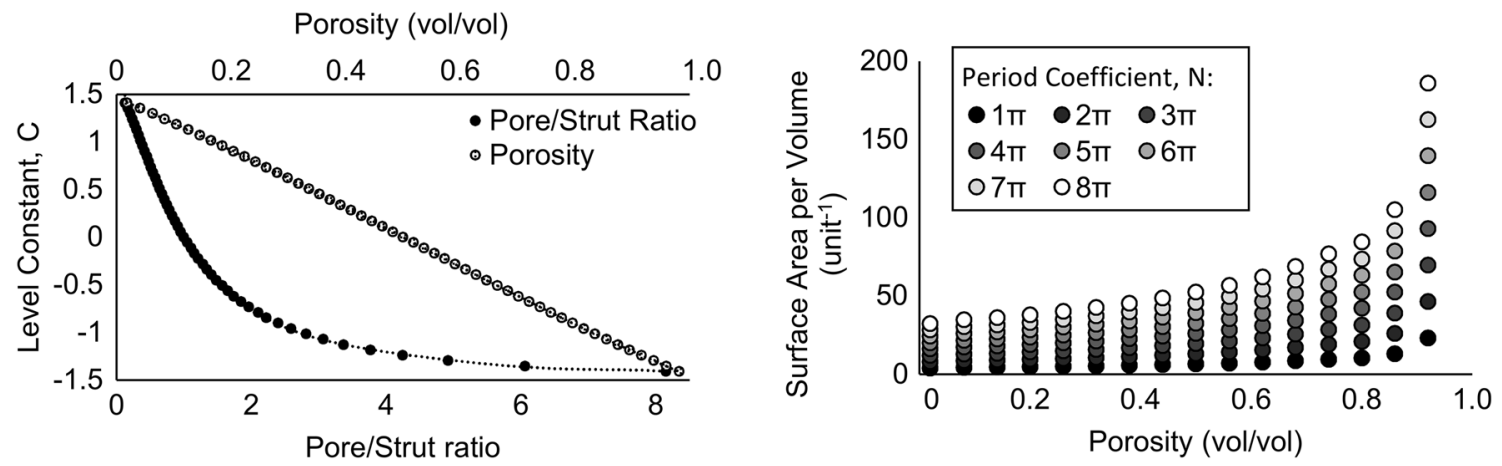

Fig. 8 (left) Level constant as a function of pore/strut ratio and porosity for gyroid shapes with polynomial fits; (right) Surface area per unit volume as a function of porosity showing increase in surface area as porosity and level constant increase 
To fully define the gyroid architecture, two of the following three input parameters must be specified: pore size, strut size, and porosity.

\section{Poly(propylene fumarate) test specimens}

\subsection{Resin formulation}

For all of the experiments, specimens were fabricated using a poly(propylene fumarate) (PPF)-based resin. PPF is a bioresorbable and photocrosslinkable polyester [1] and was synthesized via a chain-growth mechanism [32, 33]. To reduce the viscosity for $3 \mathrm{D}$ printing, $\operatorname{PPF}\left(\mathrm{M}_{\mathrm{n}}=\right.$ $1500 \mathrm{Da}, \mathrm{PDI}=1.6$ ) was diluted with diethyl fumarate (DEF, Sigma-Aldrich, St. Louis, MO) in a 1:1 ratio. Photoinitiators Irguacure 819 and Irgacure 784 (BASF, Ludwigshafen, Germany) were added in concentrations of 3.0 and $0.4 \%$ by mass, respectively. Finally, $0.7 \%$ oxybenzone (Sigma-Aldrich) was added to mitigate light scattering within layers.

\subsection{Cast PPF cylinders}

Solid cylinders were cast from PPF resin using transparent glass tubes with $4.5 \mathrm{~mm}$ inner diameter. Tubes were loaded with PPF resin and placed into a ProCure 350 UV chamber (3D-Systems, Rock Hill, SC) for $480 \mathrm{~min}$ to ensure a fully crosslinked network. After curing, PPF cylinders were removed from the glass tubes and cut to approximately $9.0 \mathrm{~mm}$ height.

\subsection{D printed PPF cylinders}

Solid cylinders with a diameter of $4.5 \mathrm{~mm}$ were additively manufactured from PPF resin using an EnvisionTEC Perfactory Micro Advantage 3D printer (EnvisionTEC, Dearborn, MI). The printer was configured with the following parameters: layer thickness $=50 \mu \mathrm{m}$, irradiance $=350 \mathrm{~mW} /$ $\mathrm{dm}^{2}$, and layer projection time $=180 \mathrm{~s}$. Cylinders were oriented vertically and at a $45^{\circ}$ angle. After printing, cylinders were immediately rinsed with acetone, $70 \%$ ethanol (v/v), and distilled water for $30 \mathrm{~s}$ each using wash bottles. Cylinders were then post-cured for $480 \mathrm{~min}$ at ambient conditions in a ProCure 350 UV chamber.

\subsection{D printed PPF porous cubes}

Porous PPF cubes were designed with $10 \mathrm{~mm}$ side length and a pore architecture consisting of $400 \mu \mathrm{m}$ struts and $1400 \mu \mathrm{m}$ pores, resulting in a designed porosity of $88.2 \%$. Porous cubes were additively manufactured from PPF resin on an EnvisionTEC Perfactory P3 Mini Multi Lens 3D printer. The build parameters were: layer thickness $=50 \mu \mathrm{m}$, irradiance $=350 \mathrm{~mW} / \mathrm{dm}^{2}$, and layer projection time $=30 \mathrm{~s}$. Porous cubes were oriented vertically, at a $45^{\circ}$ angle, and at a $90^{\circ}$ angle. After printing, porous cubes were cleaned and post-cured per the same protocol as the solid cylinders.

\section{Compression testing}

Compressive mechanical properties were evaluated by uniaxial unconfined compression on a TestResources load frame with a $250 \mathrm{lbf}$ load cell (TestResources, Shakopee, $\mathrm{MN})$. All samples were compressed at a rate of $1.0 \%$ strain per second. Young's Moduli and compressive stiffness were calculated according to the slope of the linear portion of the stress-strain curve and the yield strength was evaluated with a $0.2 \%$ offset. ANOVA methods were used to compare quantitative data across two or more groups (Minitab Inc., State College, PA, USA). Data are presented as mean \pm standard deviation from $n=6$ sample groups for solid cylinders and $n=8$ groups for porous cubes.

\section{Results and discussion}

The compressive mechanical properties of cast and additively manufactured PPF solid cylinders are shown in Fig. 10. The cast cylinders exhibited an average Young's Modulus of $593 \pm 31 \mathrm{MPa}$ and an average yield stress of $37 \pm 1 \mathrm{MPa}$. Compared to both $3 \mathrm{D}$ printed groups, the cast specimens exhibited significantly higher mechanical properties $(P<0.005)$. Neither the average Young's Modulus of the 3DP-Vertical $(481 \pm 29 \mathrm{MPa})$ and $3 \mathrm{DP}-45^{\circ}$ $(456 \pm 39 \mathrm{MPa})$ nor the average yield stress of the 3DPVertical $(29 \pm 2 \mathrm{MPa})$ and $3 \mathrm{DP}-45^{\circ}(27 \pm 4 \mathrm{MPa})$ were significantly different than each other. The drop in mechanical strength between the cast and 3D printed specimens could be due to the layer-based process with which the $3 \mathrm{D}$ printed specimens were fabricated. The lack of a measurable difference between the two 3D printed orientations is an encouraging demonstration of mechanical isotropy in layer-fabricated parts. It must be noted, however, that the 3D printed solid cylinders were fabricated with an increased exposure time of $180 \mathrm{~s}$ per layer. The long exposure time was necessary to overcome the capillary force between the part and the $3 \mathrm{D}$ printer vat, a problem common to mask projection stereolithography [34], and may serve to strengthen the bond between layers.

Porous PPF specimens which were designed with gyroid architecture and 3D printed are shown in Fig. 11. Specimens with a cubic shape were manufactured at three orientations: vertical, $45^{\circ}$, and $90^{\circ}$. In Fig. 12, the 

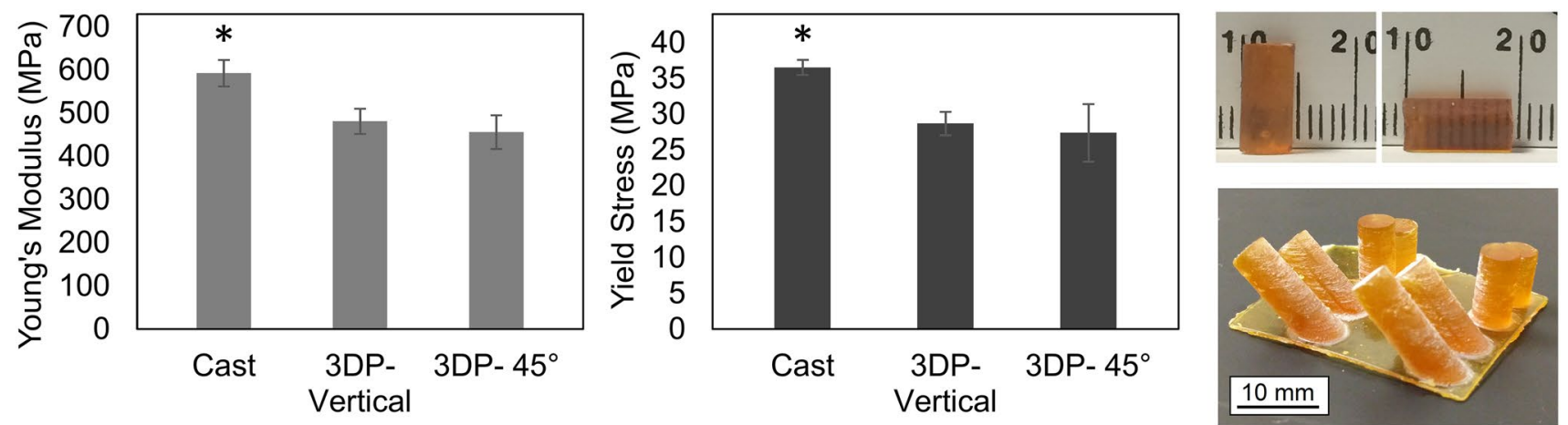

Fig. 10 Young's Modulus and yield stress of solid PPF cylinders that were cast and 3D printed at different orientations. Significance indicated refers to $P<0.005$; (upper right) photograph of cast PPF cyl- inders; (lower right) photograph of 3D printed solid PPF cylinders oriented vertically and at a $45^{\circ}$ angle
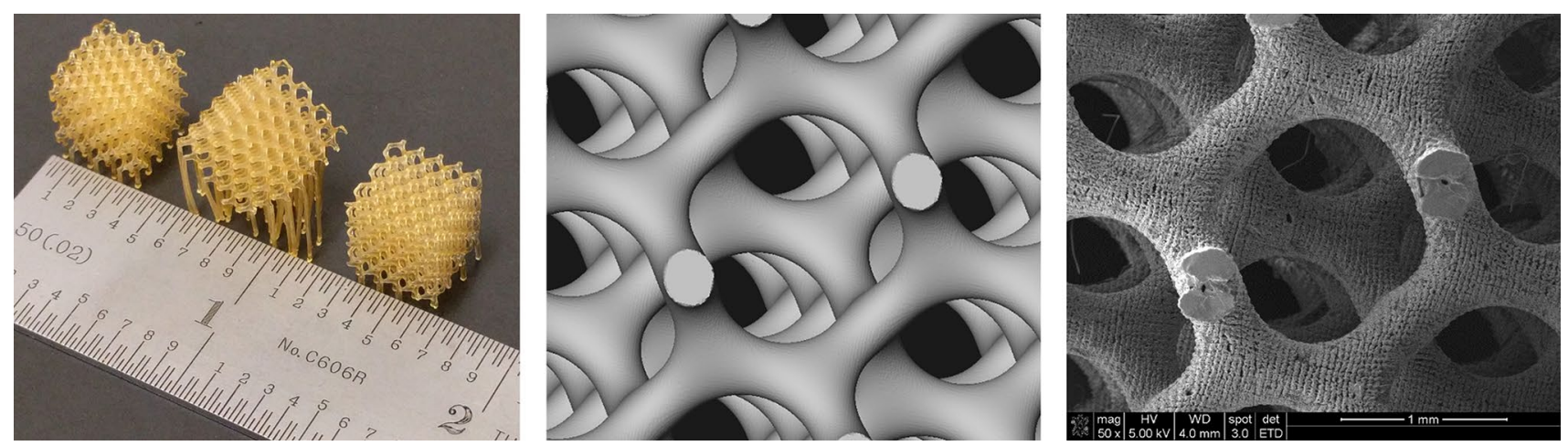

Fig. 11 (left) $10 \mathrm{~mm}$ 3D printed porous PPF cubes with $400 \mu \mathrm{m}$ struts and $1400 \mu \mathrm{m}$ pores. L to R build orientation: vertical, $45^{\circ}$, and $90^{\circ}$; $($ middle) CAD model: close-up of pore architecture; (right) SEM image of 3D printed PPF cube (scale bar $1.0 \mathrm{~mm}$ )
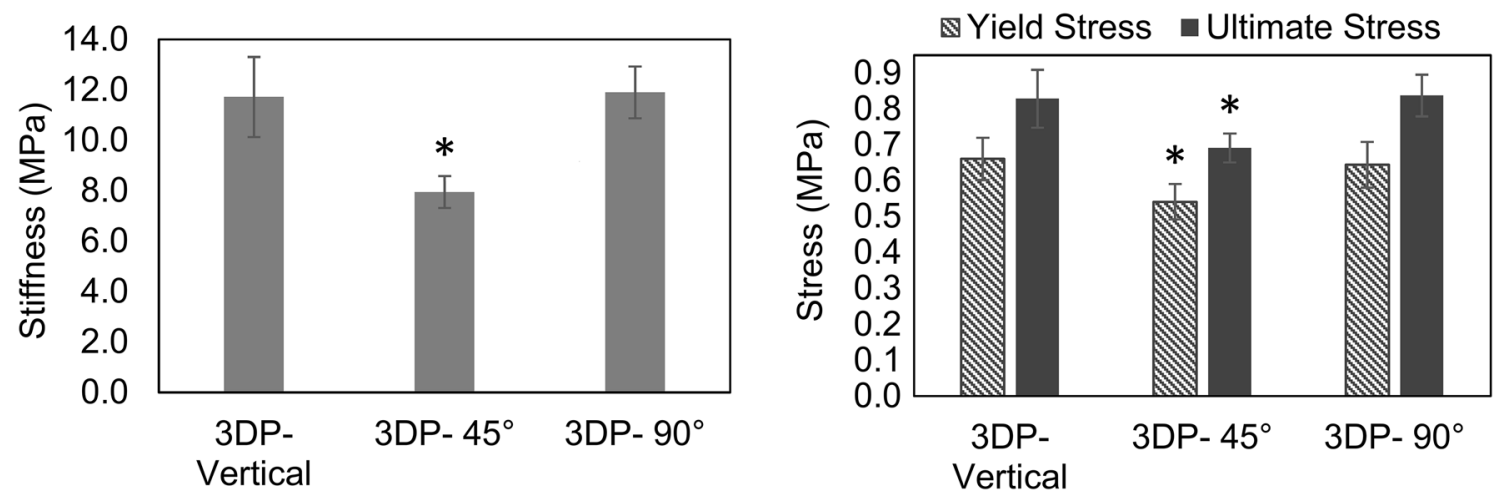

Fig. 12 Young's Modulus, yield stress, and ultimate stress of porous PPF cubes that were 3D printed at different orientations. Significance indicated refers to $P<0.005$

compressive mechanical properties of the three groups are shown. There was no significant difference found between the vertically oriented specimens and the $90^{\circ}$ oriented specimens in any metric; however, the specimens oriented at $45^{\circ}$ were significantly different in all three metrics $(P<0.005)$. With an average stiffness of $7.94 \pm 0.63 \mathrm{MPa}$, the specimens oriented at $45^{\circ}$ were approximately $30 \%$ less stiff than the other two orientations. The yield stress $(0.54 \pm 0.05 \mathrm{MPa})$ and ultimate 
Fig. 13 (left) CAD model of solid human vertebra; (right) CAD model of the same vertebra with a gyroid-type porous architecture that has $200 \mu \mathrm{m}$ struts and $700 \mu \mathrm{m}$ pores
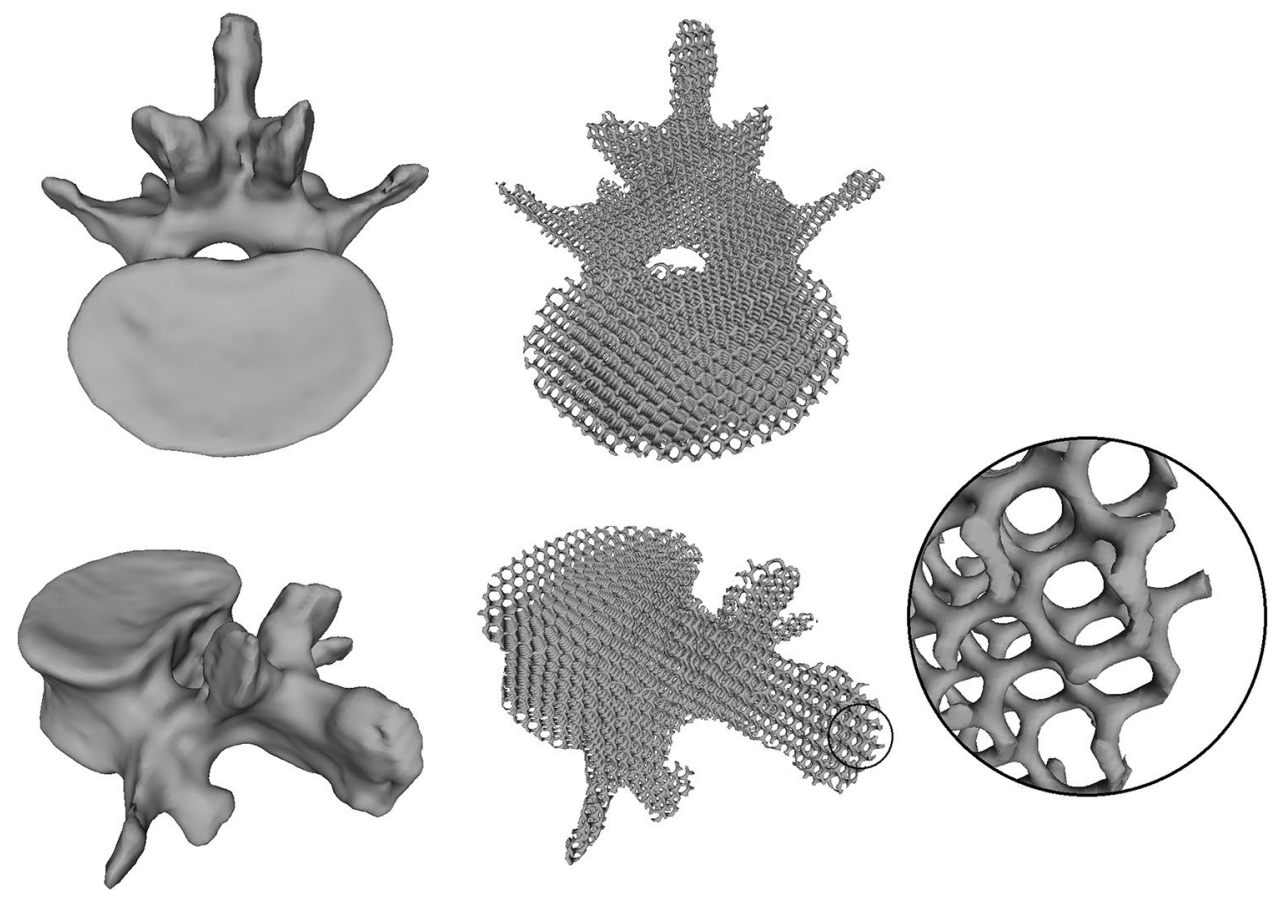

minimal surfaces have been previously linked to tissue engineering scaffolds, this is, to our knowledge, the first presentation of the gyroid surface which directly addresses feature dimensions other than percent porosity, such as strut diameter, pore size, and surface area. This design philosophy greatly increases the capacity of the gyroid as tool in tissue engineering. The ability to tailor the geometry of the solid and porous phases of a tissue engineering scaffold using virtual CAD techniques such as those presented in this paper, paired with 3D printing technologies, will empower researchers and clinicians to design and fabricate scaffolds with mechanical, degradation, and tissue-guiding properties that current implant $\mathrm{CAD}$ and conventional implant CAM methods cannot attain.

Acknowledgements The authors acknowledge partial support from the Army, Navy, NIH, Air Force, VA, and Health Affairsto support the AFIRM II effort under award No. W81XWH-14-2-0004. The US Army Medical ResearchAcquisition Activity is the awarding and administering acquisition office for award No. W81XWH-14-2-0004. Opinions, interpretations, conclusions, and recommendations are those of the authors and are not necessarily endorsed by the US Department of Defense. MLB acknowledges financial support from 21st Century Medical Technologies. We would like to thank Dr. Tanya Nocera and Mr. Robert Jones for technical assistance with mechanical analyses in the Department of Biomedical Engineering (Ohio StateUniversity, Columbus, $\mathrm{OH}$ ). We also thank Dr. Mary Beth Wade and Mr. Hernan Lara Padilla for their assistance with the preparation of figures.

\section{Compliance with ethical standards}

Conflict of interest MLB and DD have filed PCTs for the synthesis of PPF using ring opening polymerization. The PPF synthetic methods 
have been licensed to 21st Century Medical Technologies which DD and MLB have financial interests in.

\section{References}

1. Childers EP, Wang MO, Becker ML, Fisher JP, Dean D (2015) 3D printing of resorbable poly (propylene fumarate) tissue engineering scaffolds. MRS Bull 40(02):119-26

2. Lan PX, Lee JW, Seol YJ, Cho DW (2009) Development of 3D PPF/DEF scaffolds using micro-stereolithography and surface modification. J Mater Sci Mater Med 20(1):271-9

3. Sabir MI, Xu X, Li L (2009) A review on biodegradable polymeric materials for bone tissue engineering applications. J Mater Sci 1;44(21):5713-24

4. Yang S, Leong KF, Du Z, Chua CK (2001) The design of scaffolds for use in tissue engineering. Part I. Traditional factors. Tissue Eng 1;7(6):679-89

5. Hollister SJ (2005) Porous scaffold design for tissue engineering. Nat Mater 4(7):518-24

6. Kim K, Yeatts A, Dean D, Fisher JP (2010) Stereolithographic bone scaffold design parameters: osteogenic differentiation and signal expression. Tissue Eng B Rev 16(5):523-39

7. Melchels FP, Bertoldi K, Gabbrielli R, Velders AH, Feijen J, Grijpma DW (2010) Mathematically defined tissue engineering scaffold architectures prepared by stereolithography. Biomater 31(27):6909-16

8. Melchels FP, Barradas AM, Van Blitterswijk CA, De Boer J, Feijen J, Grijpma DW (2010) Effects of the architecture of tissue engineering scaffolds on cell seeding and culturing. Acta Biomater 6(11):4208-17

9. Willie BM, Petersen A, Schmidt-Bleek K, Cipitria A, Mehta M, Strube P, Lienau J, Wildemann B, Fratzl P, Duda G (2010) Designing biomimetic scaffolds for bone regeneration: why aim for a copy of mature tissue properties if nature uses a different approach? Soft Matter 6(20):4976-4987

10. Yoo D (2012) Heterogeneous minimal surface porous scaffold design using the distance field and radial basis functions. Med Eng Phys 34(5):625-39

11. Dias MR, Guedes JM, Flanagan CL, Hollister SJ, Fernandes PR (2014) Optimization of scaffold design for bone tissue engineering: a computational and experimental study. Med Eng Phys 36(4):448-57

12. Coelho PG, Hollister SJ, Flanagan CL, Fernandes PR (2015) Bioresorbable scaffolds for bone tissue engineering: optimal design, fabrication, mechanical testing and scale-size effects analysis. Med Eng Phys 37(3):287-96

13. Yousaf MN, Houseman BT, Mrksich M (2001) Using electroactive substrates to pattern the attachment of two different cell populations. Proc Nat Acad Sci 98(11):5992-6

14. Rajagopalan S, Robb RA (2006) Schwarz meets Schwann: design and fabrication of biomorphic and durataxic tissue engineering scaffolds. Med Image Anal 10(5):693-712

15. Mehdizadeh H, Brey EM, Cinar A (2014) Modeling vascularization in tissue engineering scaffolds. In: Brey EM (ed) Taylor \& Francis, London

16. Karande TS, Ong JL, Agrawal CM (2004) Diffusion in musculoskeletal tissue engineering scaffolds: design issues related to porosity, permeability, architecture, and nutrient mixing. Ann Biomed Eng 32(12):1728-43

17. Kapfer SC, Hyde ST, Mecke K, Arns CH, Schröder-Turk GE (2011) Minimal surface scaffold designs for tissue engineering. Biomaterials 32(29):6875-82
18. Yoo DJ (2011) Porous scaffold design using the distance field and triply periodic minimal surface models. Biomaterials 32(31):7741-54

19. Yoo D (2012) New paradigms in internal architecture design and freeform fabrication of tissue engineering porous scaffolds. Med Eng Phys 34(6):762-76

20. Schoen AH (1970) Infinite periodic minimal surfaces without self-intersections, NASA Techn. Rep. D-5541

21. Olmsted PD, Milner ST (1998) Strong segregation theory of bicontinuous phases in block copolymers. Macromolecules 31(12):4011-22

22. Bates FS, Hillmyer MA, Lodge TP, Bates CM, Delaney KT, Fredrickson GH. (2012) Multiblock polymers: panacea or Pandora's box?. Science 336(6080):434-40

23. Michielsen K, Stavenga DG (2008) Gyroid cuticular structures in butterfly wing scales: biological photonic crystals. J R Soc Interface 5(18):85-94

24. Saranathan V, Osuji CO, Mochrie SG, Noh H, Narayanan S, Sandy A, Dufresne ER, Prum RO (2010) Structure, function, and self-assembly of single network gyroid (I4132) photonic crystals in butterfly wing scales. PNAS 107(26):11676-81

25. Landh $\mathrm{T}$ (1995) From entangled membranes to eclectic morphologies: cubic membranes as subcellular space organizers. FEBS Lett 369(1):13-7

26. Solid State Sciences Committee (1996) Biomolecular selfassembling materials: scientific and technological frontiers. Nat Academies Press, Washington, DC

27. Von Schnering HG, Nesper R (1991) Nodal surfaces of Fourier series: fundamental invariants of structured matter. Zeitschrift für Physik B Condens Matter 83(3):407-12

28. Gandy PJ, Bardhan S, Mackay AL, Klinowski J (2001) Nodal surface approximations to the P, G, D and I-WP triply periodic minimal surfaces. Chem Phys Lett 336(3):187-95

29. Jones MW. The production of volume data from triangular meshes using voxelisation. In: Comp Graph Forum 1996 Dec 1 (vol. 15, No. 5, pp. 311-318). Blackwell Science Ltd

30. Lorensen WE, Cline HE. Marching cubes: a high resolution 3D surface construction algorithm. In: ACM siggraph comp graph 1987 Aug 1 (vol. 21, No. 4, pp. 163-169). ACM

31. Nystroem I, Udupa JK, Grevera GJ, Hirsch BE. Area of and volume enclosed by digital and triangulated surfaces. In Med Imag 2002 May 16 (pp 669-680). Int Soc Opt and Photonics

32. Luo Y, Dolder CK, Walker JM, Mishra R, Dean D, Becker ML (2016) Synthesis and biological evaluation of well-defined poly (propylene fumarate) oligomers and their use in 3D printed scaffolds. Biomacromolecules 17(2):690-7

33. Walker JM, Bodamer E, Krebs O, Luo Y, Kleinfehn AP, Becker ML, Dean D (2017) Effect of chemical and physical properties on the in vitro degradation of $3 \mathrm{D}$ printed high resolution poly (propylene fumarate) scaffolds. Biomacromolecules. doi:10.1021/acs.biomac.7b00146

34. Zhou C, Chen Y, Yang Z, Khoshnevis B (2013) Digital material fabrication using mask-image-projection-based stereolithography. Rapid Prototyp J 19(3):153-65

35. Dean D, Min KJ (2003) Deformable templates for preoperative computer-aided design and fabrication of large cranial implants. In Inter Congress Ser (vol. 1256, pp. 710-715). Elsevier

36. Dean D, Min KJ, Bond A (2003) Computer aided design of large-format prefabricated cranial plates. J Craniofac Surg 14(6):819-32

37. Wallace J, Wang MO, Thompson P, Busso M, Belle V, Mammoser N, Kim K, Fisher JP, Siblani A, Xu Y, Welter JF (2014) Validating continuous digital light processing (cDLP) additive manufacturing accuracy and tissue engineering utility of a dyeinitiator package. Biofabrication 6(1):015003 